\title{
WRD: an approach for evaluating the performance of training program in virtual reality
}

\author{
Liudmila Steshina, Igor Petukhov, and Andrey Glazyrin \\ Department of Radio Technical Volga State University of Technology Yoshkar-Ola, Russia
}

\begin{abstract}
Keywords: Virtual reality, Virtual subjectivities, Human-computer interaction, Training simulator, Fuzzy model.
\end{abstract}

\begin{abstract}
The paper presents a new approach for evaluating the performance of the training program in virtual reality. The training process is described through three main steps: watching, recalling and doing (WRD). Each step is related to the sensor-motoric layer. The watching is related to the sensory layer, which accumulates the visual information from virtual reality. After then, that visual information will source for recalling the previous experiences followed by the decision-making using the cognitive layer. Finally, the decision-making activates the motor layer in form doing. The WRD approach uses the tests for the measuring of motor and sensory layers. The tests describe typical model of reaction for operator and activate motor and sensory layers as in real professional case. The cognitive layer may be measured with EEG. At the same time, the continuous generation of training cases may help to saturate the knowledge base. For this reason, the fuzzy model based on the generation of training cases was developed. The analysis of experimental data showed the decreasing of time delay by $83 \%$. Moreover, it was shown the decreasing of rejection from horizontal and vertical axes in pixels by $62.7 \%$ and decreasing of time expected by $57 \%$ for motor tracking. The WRD approach may help to evaluate training program with evaluating sensory, cognitive and motor layer without developing prototype training simulator.
\end{abstract}

\section{Introduction}

Training in virtual reality (VR) has been becoming standard in different industries. Almost any technological process may be placed inside the virtual environment (VE), which is safe for humans. Moreover, it was shown the effectiveness in memorizing [1] and motor training [2] using VR. It may be related to the presence (immersion) is the main feature of VR. The presence may activate an artificial stimulus as if it was real. From this reason, the realism of training for technological operation based on presence is better than training in desktop or other environments.

It was shown that VE has an influence on emotional states and stress resistance [3]; thus, this one could activate the corresponding behavior like in the real world [4]. The analysis 
mentioned above shows the potential of VR in the context of increasing the effectiveness of learning and simulation training.

There are many approaches to developing and performance evaluation VR for training. Each approach is related to specific of the training process and central elements are included inside the training models such as avatars or mannequins. Moreover, many performance evaluation methods in virtual environment (VE) are used such as Fitt's law, heuristic evaluations, cognitive walkthrough, and other performance models [5]. For the above-mentioned reasons, the choice of approach for developing VR for training may face difficulties.

The rest of the paper is structured as follows. Section "Related works" describes the main problems of human-computer interaction within VR and covers some related works in this area. Section "Materials and methods" mentions the WRD approach, which is used for evaluating the performance in VR and the fuzzy model based on changing the complexity is presented. Section "Results and Discussions" concludes experimental results WRD approach.

\section{Related works}

There are different approaches in designing of immersion environment for operator training simulator and it can be related by professional specific of operator's acts.

It's important to consider psychophysiological features in VR interaction; therefore user-centered approach should be getting important attention. The user-centered approach (UCA) considers human as complex system takes into consideration perceptual features, cognitive and motor skills in process of interaction with VE [6].

At the same time, it was shown importance of virtual subjectivities in VR [7]. There are three components: physical interface, logical interface and mapping between physical and logical interfaces [7]. The user interacts to VR through those components. Moreover, virtual subjectivity may be related to mapping based on logical and physic interfaces. That is why it needs to consider the process of perception activity.

Moreover, the virtual subjectivities impact of the environment itself seems to be mapping or correct association between user's moving and view rendering. In the same time, it was shown a sequence acts in VE [8] and network model of operator's processing information based on perceptual, cognitive and motor functions [9].

It is important to extract a human feature, which affects the performance of the VE. For example, in the Conceptual VR Model (CVRM), the user handles effectors (shell, fixture, and appliance) from VR, which reduce feedback in the form of sensory stimuli. Consequently, human interaction with VR it is needed finds out the mapping of the virtual effectors and the perceptual system of the participant. So, the visual perceptual system is linked with visual display such as orientation in time and space.

\section{Materials and methods}

Therefore, each operator action may be presented in form of sensor-motoric reaction. Firstly, the operator watches currently situation, which is related to orientation and adaptation inside VR during training. Before training acts the operator makes a decision based on recalling a previous experience, how he/she will do the task more efficiently. Finally, operator uses motor program for current task. Therefore, we have three main steps in training process: watching + recalling + doing (WRD).

The key idea of WRD approach is the decomposition of sensor-motoric process into three layers. The sensory layer (watching) covers visual capturing of important information 
from environment. After the accumulation of important information occurs the querying to knowledge base (recalling) for decision-making in current situation. This process is performed on cognitive layer. If knowledge base contains same professional case then scheme of motor reaction (doing) is retrieved. In the same time, if knowledge base doesn't contain current professional case then knowledge base is saturated an unknown situation. Finally, operator activates motor program based on decision-making in current situation from cognitive layer.

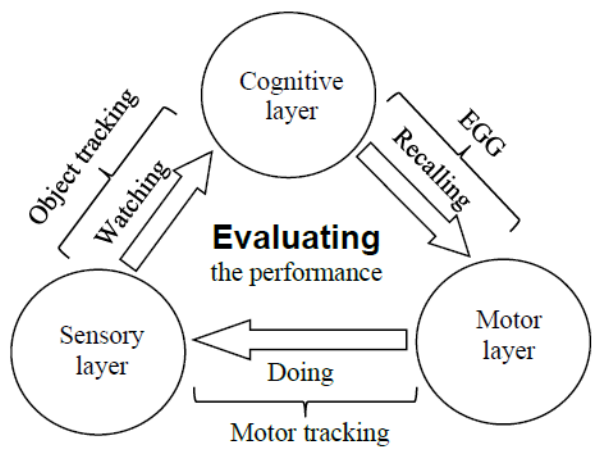

Fig. 1. WRD approach with decomposition training process into three main steps: watching, recalling and doing.
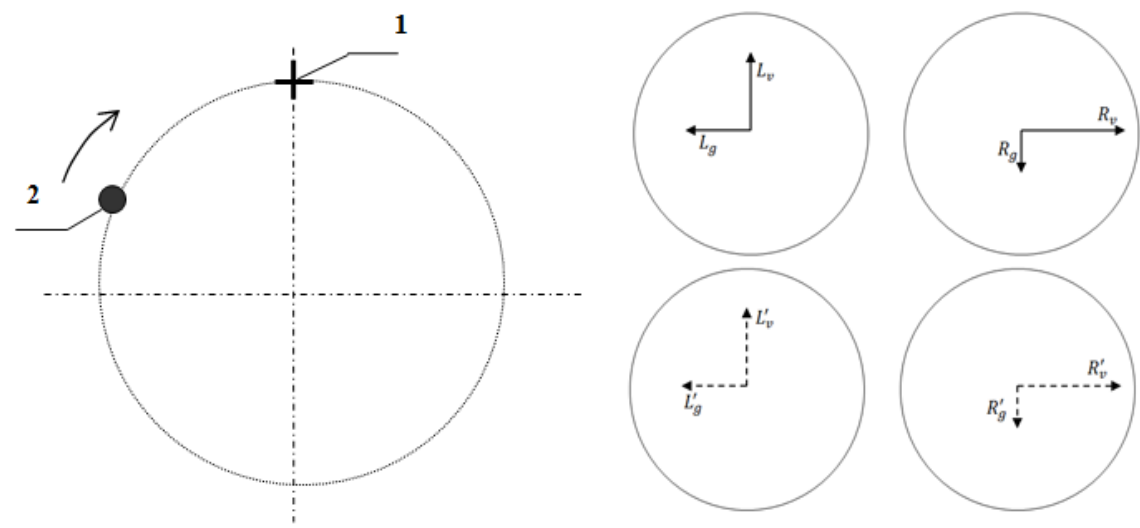

Fig. 2. Left: Object tracking. The cross labeled "1" is breakpoint for moving object labeled "2". Right: Motor tracking. Top cycles are etalons with two vectors: $L_{v}, L_{g}$ (for left hand) and $R_{v}, R_{g}$ (for right hand). Bottom cycles are user track with two vectors: $L_{v}^{\prime}, L_{g}^{\prime}$ (for left hand) and $R_{v}^{\prime}, R_{g}^{\prime}$ (for right hand).

We believe on sensory layer may be used test so called object tracking, pic.1 (Left). The object tracking activates sensory organs in same manner as sensor-motoric reaction for typical operator task. Operator should press a selected key for stopping moving circle and press same key once again for restart moving. Stopped object should be placed as close as possible to cross, so delta between stopped object and cross is time delay (sec.). Time delay for fuzzy model is input parameter. The output parameter is object velocity $(\mathrm{rad} / \mathrm{sec})$.

For estimate of performance on motor layer may be used test "motor tracking", pic.2. (Right)

Motor tracking activates sensory organs in same manner as sensor-motoric reaction for typical operator task in form rejection from a selected etalon (horizontal and vertical axes). Input parameter is time expended (sec) and maximum rejection from a selected etalon (pixels). The output parameter is sensitivity tracking. For "Motor tracking" used two 
standard joysticks.

On cognitive layer, the brain activity may be measured using EEG. The EEG is tool for objective control of electric signal from brain domains in $\mathrm{mkV}$. At the same time, the brain activity in training sessions should rise because it is important to accumulate new training cases. The brain activity should fall during control session because it indicates knowledge base is saturated. That is why the continuous generation of training case may help to saturate the knowledge base. Therefore, the fuzzy model based on the generation of training cases was developed.

The WRD approach may help to evaluate training program with evaluating sensory, cognitive and motor layer without developing prototype training simulator.

Let define fuzzy model based on special tests. The special tests are defined $-T_{l}, T_{2}, \ldots, T_{t}$, then $t$ - count of tests. For each test $-T_{t}$ are defined test results $-r_{l}, r_{2}, \ldots, r_{l}$, then $l-$ count of test result and output variables are complexity for each tests $-w_{l}, w_{2}, \ldots, w_{h}$, then $h-$ count of output variables. Therefore, for each input variables and each output variables are defined fuzzy sets:

$$
\begin{aligned}
& R_{l}=\left\{\left(\mu_{R_{l}}\left(r_{l}\right), r_{l}\right)\right\}, \forall r_{l} \in R_{l}, \\
& W_{h}=\left\{\left(\mu_{W_{h}}\left(w_{h}\right), w_{h}\right)\right\}, \forall w_{h} \in W_{h},
\end{aligned}
$$

then $\mu_{R_{l}}\left(r_{l}\right), \mu_{W_{h}}\left(w_{h}\right)$ - triangle membership functions.

The choosing of mentioned triangle membership functions are related to research psychophysiological reactions of operators such as the choosing of specific interval values for training operator $[\mathrm{xx}]$. Set of rules for fuzzy inference:

RULE $^{(c)}$ : IF $r_{l}$ это $R_{1}^{\mathrm{c}}$ AND $r_{2}$ это $R_{2}^{\mathrm{c}} \ldots$ AND $r_{l}$ это $R_{l}^{\mathrm{c}}$ THEN $w_{l}$ это $W_{1}^{\mathrm{c}}$ AND $w_{2}$ это $W_{2}^{\mathrm{c}} \ldots$ AND $w_{h}$ это $W_{h}^{\mathrm{c}}\left(F_{\mathrm{c}}\right)$, then $c$ - count of fuzzy rule and $l \neq h, F_{\mathrm{c}}-$ weight of rule.

For fuzzy procedure was used the Mamdani Fuzzy Method. The base of rule and membership functions is presented at pic.3.

The fuzzy model based on changing of complexity. If the operator made maximum errors in a training session then the test generated the simple training case with lower complexity. At the same time, if the operator made minimum errors in a training session then the test generated a simple training case with higher complexity.

\section{Results and discussions}

Two groups (35 each) of students were formed. The first group labeled "team 1" (students used tests without fuzzy model i.e. stationary training session) and second group labeled "team 2" (students used tests with fuzzy model). For control of training session were used the tests with increased complexity without fuzzy model.

The obtained experimental results are summarized at table 1. In training session, the performance was decreased for "team 2", but in control session performance was significantly increased for "team2" in form the decreasing of time delay by $83 \%$ for test "Moving object". On the contrary, in control session performance was significantly decreased for "team 1". In control session the performance was significantly increased for "team2" in form decreasing of rejection from horizontal and vertical axes in pixels by $62.7 \%$ and decreasing of time expected by $57 \%$ for test "Motor tracking".

For the measuring performance on cognitive layer used EEG during training and control sessions for moving objects, pic.3. The obtained experimental results in form EEG signals $(\mathrm{mkV})$ are shown in figure 3. The cognitive capacity for "Team 2" $(9-12.5 \mathrm{mkV})$ is higher than "Team 2" (5-7 mkV) in training session. In the same time, cognitive capacity for "Team 1" (7-12 mkV) is higher than "Team 1" (5-6 mkV) in control session. It may be 
related to the increased brain activity in training session for reason changing complexity for training program.

The fuzzy model for sensory layer (moving object)
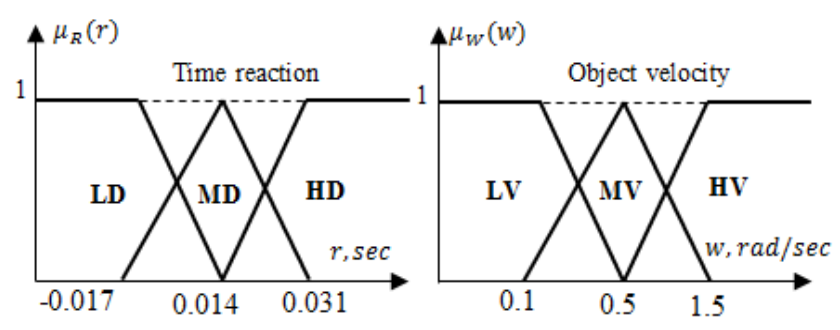

IF $r$ IS LD THEN $w$ IS LV;
IF $r$ IS MD THEN $w$ IS $\mathbf{~ M V ;}$
IF $r$ IS HD THEN $w$ IS HV;

LD - low time delay;

MD - middle time delay;

HD - high time delay;

LV - low velocity;

MV - middle velocity;

HV - high velocity.

The fuzzy model for motor layer (motor tracking)
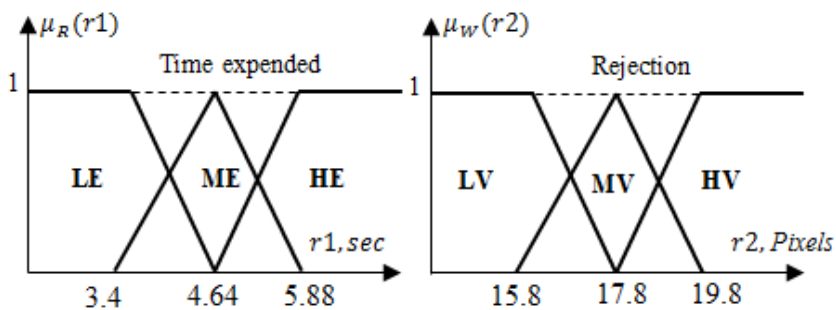

\begin{tabular}{|c|c|c|}
\hline \multicolumn{2}{|c|}{ IF } & THEN \\
\cline { 1 - 2 }$r_{1}$ IS & $r_{2}$ IS & IS \\
\hline LE & LV & HS \\
\hline LE & MV & HS \\
\hline LE & HV & LS \\
\hline ME & LV & HS \\
\hline ME & MV & MS \\
\hline ME & HV & HS \\
\hline HE & LV & MS \\
\hline HE & MV & \\
\hline HE & LV & \\
\hline
\end{tabular}

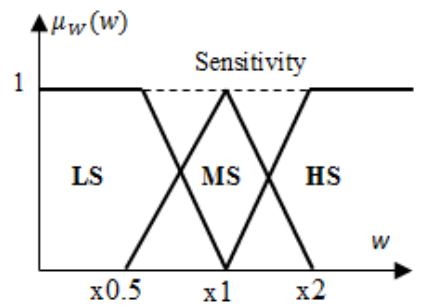

LE - low time expended;

ME - middle time expended;

HE - high time expended;

$\mathbf{L V}$ - low velocity;

MV -middle velocity;

HV - high velocity;

LS - low sensitivity;

MS - middle sensitivity;

HS - high sensitivity.

Fig. 3. Fuzzy model for sensory layer and motor layer.

We believe the increased brain activity in training sessions for "Team 2" is due to the fact that saturation of knowledge base has occurred. The continuous generation of new professional cases may accumulate of knowledge for future decision-making.

Thereby, experimental results may be related to the uniform actions based on static training program for "team 1". In the same time, "team 2 " used the different models of reactions for changing training program based on the changing of test complexity. Therefore, when "team 2" faced with new training case, it was not the problem in choosing the relevant models of reactions for current the test. On the contrary, the "team 1" was adapted to single training case with constant complexity. For this reason, when "team 2" faced with new training case, it was the problem in choosing the relevant models of reactions for current test.

Thereby, the fuzzy model based on the changing of test complexity shown the increasing of operator performance in the form of decreasing time delay in test "moving object" and the rejection from horizontal and vertical axes in test "motor tracking". Moreover, the research of the cognitive layer based on EEG showed the efficiency of the changing training program with the generation of different training cases. 


\section{Conclusions and future works}

The WRD approach for evaluated the performance of training in VR is presented. There are three main steps inside VR during training. Firstly, the operator watches currently situation, after this operator makes a decision based on recalling a previous experience, how he/she will do a task more efficiently. Finally, operator uses motor program for current task. Therefore, we have three main steps in training process: watching + recalling + doing (WRD). Moreover, each step may be linked to the senso-motoric reaction.

Table 1. Experimental results for moving object and motor tracking.

\begin{tabular}{|c|c|c|c|}
\hline \multicolumn{3}{|c|}{ Moving object in seconds } \\
\hline Training session & \multicolumn{2}{c|}{ Control session } \\
\hline Team 1 & Team 2 & Team 1 & Team 2 \\
\hline$M x(1)=0.18$ & $M x(2)=0.14$ & $M x(1)=0.28$ & $M x(2)=0.05$ \\
\hline$|M x(1)-M x(2)|=0.04(21 \% \downarrow)$ & $|M x(1)-M x(2)|=0.23(83 \% \downarrow)$ \\
\hline \multicolumn{4}{|c|}{ Motor tracking for rejection from horizontal and vertical axes in pixels } \\
\hline Training session & Control session \\
\hline Team 1 & Team 2 & Team 1 & Team 2 \\
\hline$M x(1)=23.31$ & $M x(2)=21.14$ & $M x(1)=28.5$ & $M x(2)=10.6$ \\
\hline$|M x(1)-M x(2)|=2,17(12 \% \downarrow)$ & $|M x(1)-M x(2)|=17,9(62.7 \% \downarrow)$ \\
\hline \multicolumn{4}{|c|}{ Motor tracking for time expected in seconds } \\
\hline Training session & Tentrol session \\
\hline Team 1 & $M x(2)=24.07$ & $M x(1)=26.26$ & $M x(2)=11.13$ \\
\hline$M x(1)=13.71$ & $|M x(1)-M x(2)|=15.14(57 \% \downarrow)$ \\
\hline$|M x(1)-M x(2)|=-10.36(43 \% \uparrow)$ &
\end{tabular}

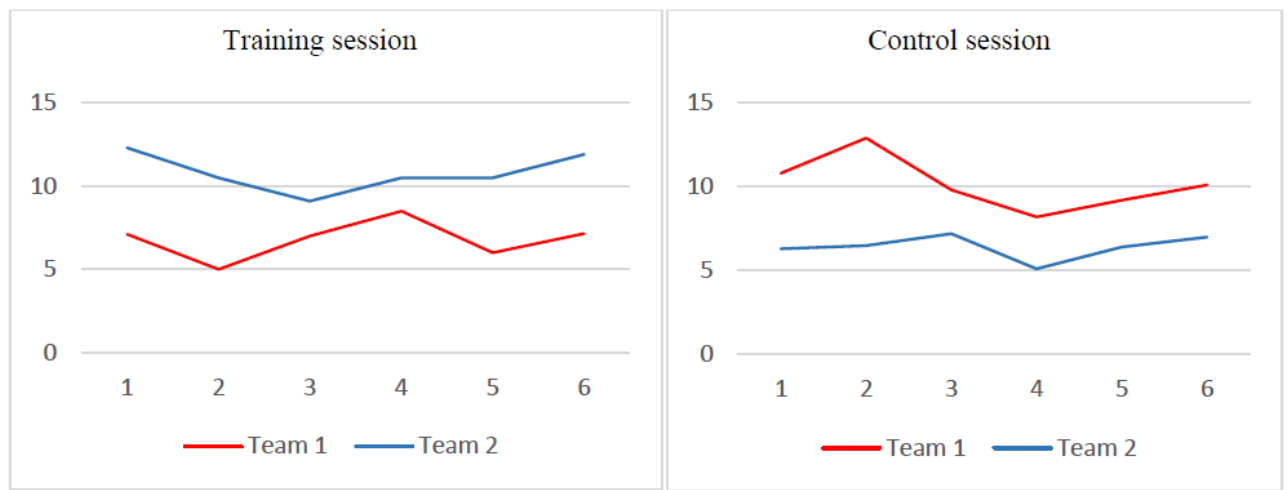

Fig.3. EEG signals for "team 1" (blue line) and "team 2" (red line). The horizon axis is number of student and vertical is values of microvolts EEG.

The watching may be described through the sensory layer, which picks up visual information from the surrounded environment such as VE. After then, that visual information will source for recalling the previous experiences followed by the decisionmaking using the cognitive layer. The decision-making activates motor program (doing) on motor layer in form a set of muscle motions.

Thereby, for WRD approach the performance inside training VR is summarized from performance of each layer. For example, motor or sensory layers may be evaluated using special tests, which activate the sensor-motoric reaction for the appropriate operator task. The evaluating the performance on the cognitive layer is important step in WRD approach, because continuous brain activity may result the tension and fatigue. Therefore, it needs to measure the brain activity with EEG during executing tests on sensory and motor layers. 
We believe the brain activity in training sessions should rise because it is important to accumulate new training cases. The brain activity in control sessions should fall because it indicates knowledge base is saturated. The continuous generation of training case may help to saturate the knowledge base. Therefore, the fuzzy model based on the generation of training cases was developed. The key idea is the changing complexity of tests. The comparison based on WRD approach. Moving object and motor tracking (rejection from horizontal and vertical axes) were selected.

The experimental results are presented. In training session, the performance was decreased for "team 2" (learning without fuzzy model), but in control session performance was significantly increased for "team 2" in comparison with "team 1" (learning with fuzzy model). The analysis of experimental data showed the decreasing of time delay by $83 \%$ for "team 1". It was shown for "team1" the decreasing of rejection from horizontal and vertical axes in pixels by $62.7 \%$ and decreasing of time expected by $57 \%$ for motor tracking. Accordingly, using fuzzy model shown the increasing the performance for operator task.

Future work will focus on using the WRD approach for developing a forest machine simulator based on VR. The fuzzy model in the training simulator will include the environment parameters, which are changing its characteristics. Those characteristics are influenced on the complexity of executing operator tasks.

The results of this study were obtained with the support of Grant No. 25.1095.2017/4.6.

\section{References}

1. Krokos, E., Plaisant, C., \& Varshney, A. (2019). Virtual memory palaces: immersion aids recall. Virtual Reality, 23(1), 1-15.

2. Maggio, M. G., Russo, M., Cuzzola, M. F., Destro, M., La Rosa, G., Molonia, F., \& Calabrò, R. S. (2019). Virtual reality in multiple sclerosis rehabilitation: A review on cognitive and motor outcomes. Journal of Clinical Neuroscience.

3. Fabri, M., Moore, D. J., \& Hobbs, D. J. (1999, March). The emotional avatar: Nonverbal communication between inhabitants of collaborative virtual environments. In International gesture workshop (pp. 269-273). Springer, Berlin, Heidelberg.

4. C. Boletsis, "The New Era of Virtual Reality Locomotion: A Systematic Literature Review of Techniques and a Proposed Typology," Multimodal Technologies and Interaction, 2017, vol. 1, № 4, 24.

5. Bowman, D. A., Coquillart, S., Froehlich, B., Hirose, M., Kitamura, Y., Kiyokawa, K., \& Stuerzlinger, W. (2008). 3d user interfaces: New directions and perspectives. IEEE computer graphics and applications, 28(6), 20-36.

6. Petukhov I. et al. Design Model of a Training Simulator in Virtual Reality// The Fifth International Conference on Fundamentals and Advances in Software Systems Integration, FASSI 2019 - P.1-7.

7. Parés, N., \& Parés, R. (2006). Towards a model for a virtual reality experience: the virtual subjectiveness. Presence: Teleoperators and Virtual Environments, 15(5), 524538.

8. Jerald, J. (2015). The VR book: Human-centered design for virtual reality. Morgan \& Claypool.

9. Liu, Y., Feyen, R., \& Tsimhoni, O. (2006). Queueing Network-Model Human Processor (QN-MHP): A computational architecture for multitask performance in human-machine systems. ACM Transactions on Computer-Human Interaction (TOCHI), 13(1), 37-70. 